\title{
High Pressure Dielectric Studies of a Substance with the Smectic $A_{1}$ Phase
}

\author{
Stanisław Urban and Albert Würflinger ${ }^{a}$ \\ Institute of Physics, Jagellonian University, Reymonta 4, 30-059 Cracow, Poland \\ ${ }^{a}$ Physical Chemistry II, Ruhr University, D-44780 Bochum, Germany \\ Reprint requests to Prof. S. U.; Fax: 0048-12-633-7086; E-mail: ufurban@cyf-kr.edu.pl
}

Z. Naturforsch. 54a, 455-458 (1999); received July 12, 1999

The results of dielectric studies of 5- $n$-hexyl-2-(4'-isothiocyanato)-1,3-dioxane (6DBT) in the smectic $\mathrm{A}_{1}$ phase at pressures up to $150 \mathrm{MPa}$ and temperatures up to $340 \mathrm{~K}$ are presented. The low frequency relaxation time $\tau_{\|}(p, T)$ yields the activation volume $\Delta^{\#} V_{\|}=R T\left(\partial \ln \tau_{\|} / \partial p\right)_{T}$ and activation enthalpy $\Delta^{\#} H_{\|}=R\left(\partial \ln \tau_{\|} / \partial T^{-1}\right)_{p}$. The calculated values of these parameters are compared with those obtained recently for $n$-octyl-cyanobiphenyl (8CB) forming the smectic $\mathrm{A}_{\mathrm{d}}$ phase. In the case of 6DBT both these quantities are practically constant, whereas those for $8 \mathrm{CB}$ decrease, when going away from the phase transitions isotropic - smectic $A_{1}(6 D B T)$ or nematic - smectic $A_{d}(8 C B)$. These differences support our earlier conclusion that increase of pressure leads to a breaking of the antiparallel associations of cyanobiphenyl molecules in the smectic as well as in the nematic phases.

Key words: Liquid Crystal; Smectic A, Dielectric Properties, High Pressure.

\section{Introduction}

Our recent high pressure (h.p.) studies of the low frequency (l.f.) dielectric relaxation process in the smectic A (Sm A) phase concerned liquid crystalline substances with the cyano $(-\mathrm{CN})$ group at the terminal position. These substances were: 4- $n$-octyloxy- and 4- $n$ octyl-cyanobiphenyl (8OCB and 8CB) [1-3]. They form the so-called $\mathrm{Sm} \mathrm{A}_{d}$ phase in which the layer thickness $d$ does not correlate with the molecular length $l$. In the case of the above mentioned substances the ratio $d / l \approx 1,4[4]$ means that the molecules partly overlap within the layer. Some peculiar behaviour of the activation parameters derived from the h.p. dielectric relaxation studies of $8 \mathrm{OCB}$ and $8 \mathrm{CB}$ were interpreted by us as the result of breaking of antiparallel association of the molecules (the same concerns the nematic phase as well) [1-3]. For the present studies we have chosen a substance with the isothiocyanato (-NCS) terminal group which forms exclusively the $S m A_{1}$ phase [5] for which $d / l$ is close to unity [5-7]. Thus, one would expect some differences in the influence of the pressure on the parameters characterizing the molecular rotations around the short axes in comparison with those observed for the cyano-compounds.

\section{Experimental}

The substance studied, 5- $n$-hexyl-2-(4'-isothiocyanato)-1,3-dioxane (6DBT)

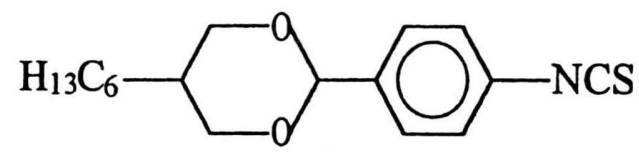

belongs to the $n \mathrm{DBT}$ homologous series in which the compounds with $n$ from 4 to 12 exhibit the smectic A polymorphism only [6,7]. 6DBT has the broadest range of the SmA phase between $307 \mathrm{~K}$ (melting) and $352 \mathrm{~K}$ (clearing). Its $(p, T)$ phase diagram was not established yet. In the present study only a rough establishment of the smectic A - crystalline transition was possible: $T_{\mathrm{SmA}-\mathrm{Cr}} \approx 307+0.19 p$ [MPa]. The experimental conditions for dielectric measurements were the same as in [2]. The measurements of the complex dielectric permittivity, $\varepsilon^{*}=\varepsilon^{\prime}-i \varepsilon^{\prime \prime}$, were performed on decreasing the pressure at constant temperature. For safety reasons the measurements could not be extended beyond $150 \mathrm{MPa}$ and $350 \mathrm{~K}$. Therefore, no spectrum for the isotropic phase could be measured due to the high temperature of the clearing point.

\section{Results}

Typical relaxation spectra measured at one pressure and different temperatures are presented in Fig. 1 in the 


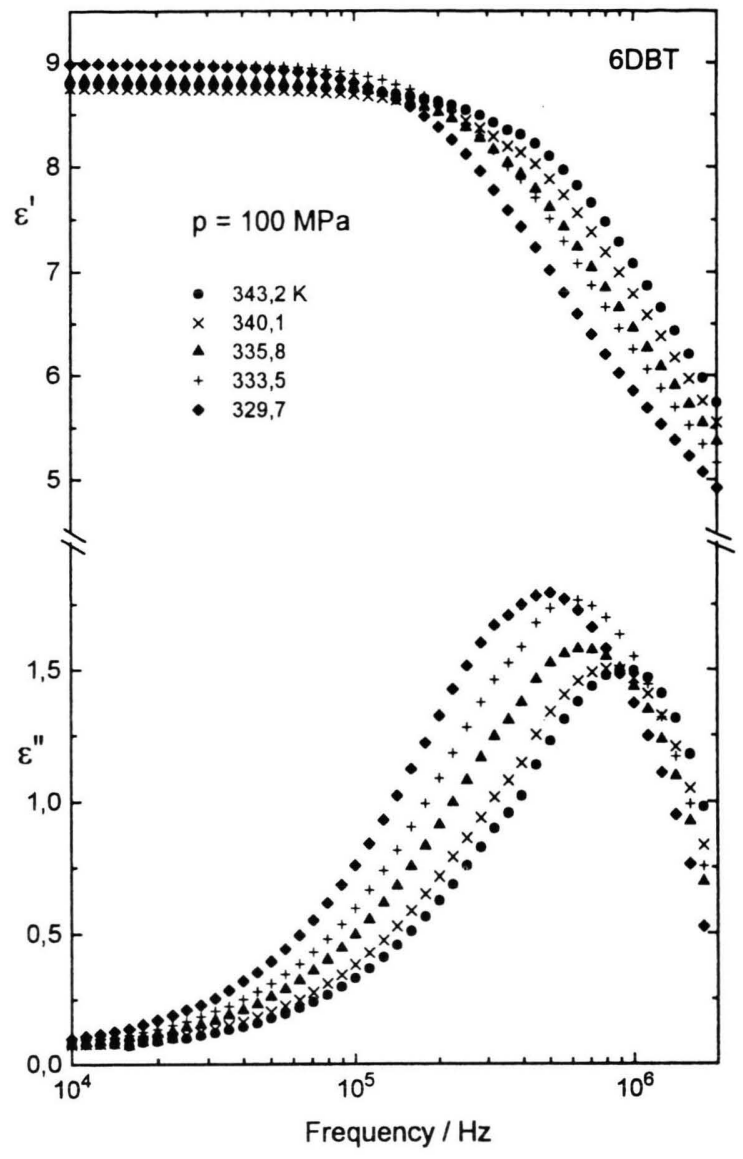

Fig. 1. Dispersion, $\varepsilon^{\prime}(v)$, and absorption, $\varepsilon^{\prime \prime}(v)$, spectra collected at one pressure $(100 \mathrm{MPa})$ and different temperatures of the Sm A phase of 6DBT.

form of the dispersion, $\varepsilon^{\prime}(v)$, and absorption, $\varepsilon^{\prime \prime}(v)$, spectra. Due to some bad contacts between the electrodes in the autoclave, caused by the pressurizing medium (silicon oil), the measurements had to be limited to ca. $2 \mathrm{MHz}$. The spectra can be described very well by the Debye equation

$$
\frac{\varepsilon^{*}-\varepsilon_{\infty}}{\varepsilon_{\mathrm{s}}-\varepsilon_{\infty}}=\frac{1}{1+i \omega \tau},
$$

where $\varepsilon_{\mathrm{s}}$ and $\varepsilon_{\infty}$ are the static and high frequency permittivities, respectively, and their difference the dielectric increments, characterize the strength of a given relaxation process. The pressure dependence of both permittivities at several temperatures within the SmA phase of 6DBT is presented in Figure 2. The relaxation times $\tau_{\|}$ were calculated from the frequency corresponding to the

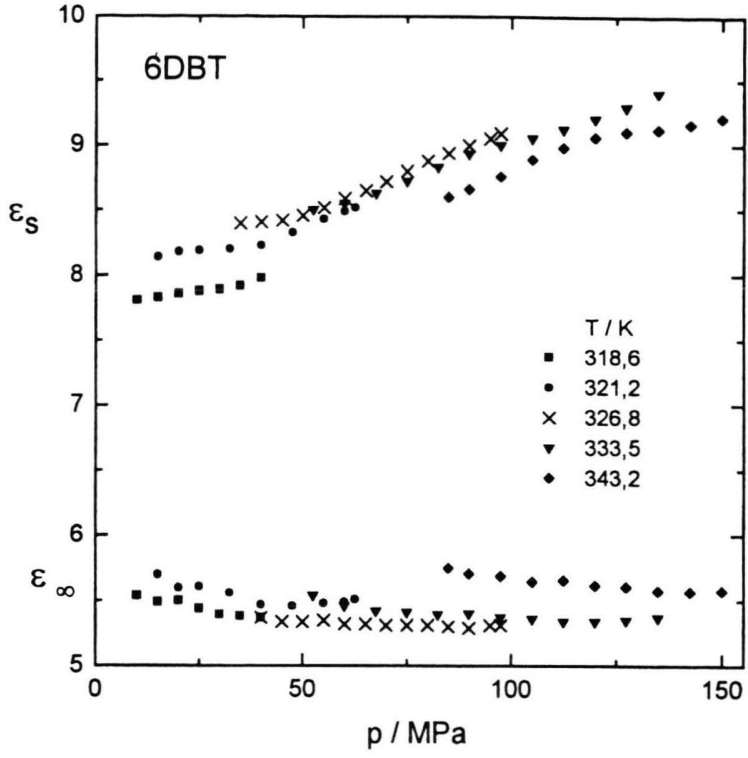

Fig. 2. Pressure dependence of the static $\varepsilon_{\mathrm{s}}$ and high frequency $\varepsilon_{\infty}$ permittivities measured at several isotherms of the Sm A phase of 6DBT.

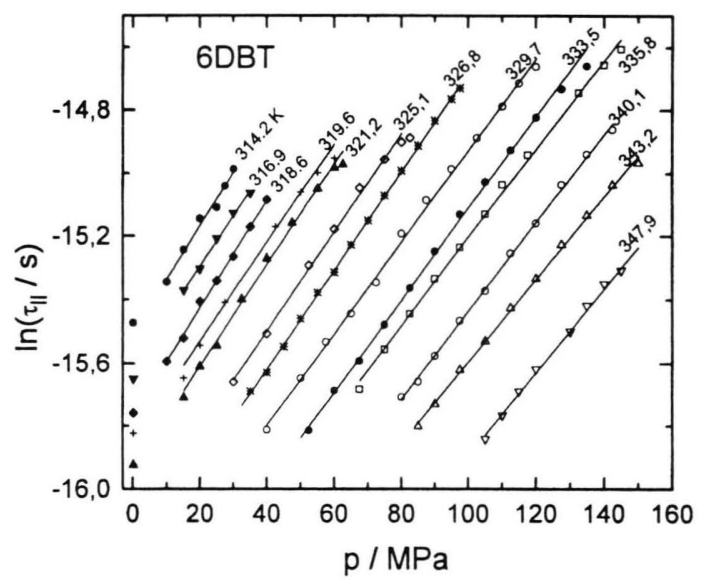

Fig. 3. Pressure dependence of the relaxation times at all studied isotherms of the Sm A phase of 6DBT.

maximum of losses $v_{\mathrm{m}}: \tau_{\|}=1 /\left(2 \pi v_{\mathrm{m}}\right)$. They are presented in semilogarithmic scale in Figure 3. Using these data, the activation parameters are derived. The slope of the curves in Fig. 3 defines the activation volume: $\Delta^{\#} V_{\|}$ $=R T\left(\partial \ln \tau_{\|} / \partial p\right)_{T}$. As can be seen in the figure, the points are well placed on the straight lines in a broad range of the pressure values. The results of calculations are presented in Fig. 4 vs. temperature. Taking the relaxation times at constant pressure one can calculate the activa- 


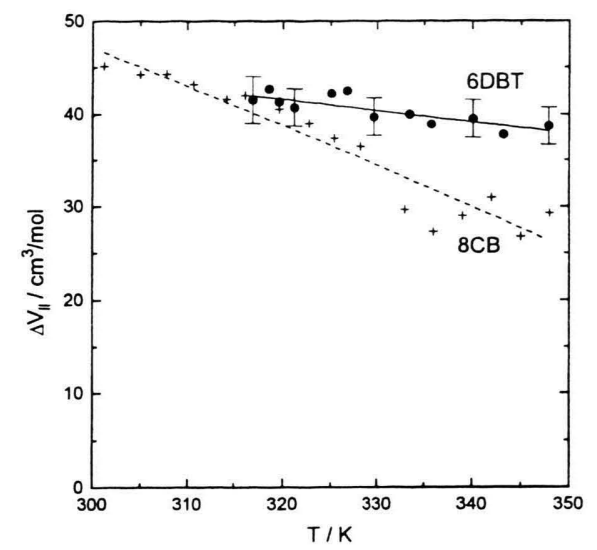

Fig. 4. Activation volume as a function of temperature of the $\mathrm{Sm}$ A phase of 6DBT. For comparison the results for 8CB taken from [2] are shown (crosses).

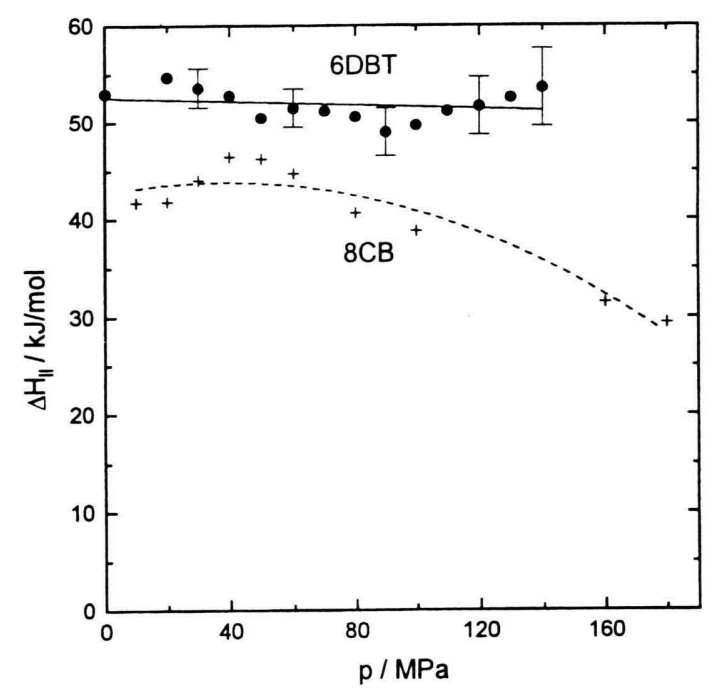

Fig. 5. Activation enthalpy as a function of pressure of the Sm A phase of 6DBT. For comparison the results for 8CB taken from [2] are shown (crosses).

tion enthalpy $\Delta^{\#} H_{\|}=R\left(\partial \ln \tau_{\|} / \partial T^{-1}\right)_{p}$, which is plotted vs. pressure in Figure 5.

\section{Discussion}

The dielectric studies of the $n \mathrm{DBT}$ compounds at ambient pressure exhibited the appearance of two relaxation processes $[7,8]$. They were attributed to the molecular rotations around the short axes (low frequency process) and around the long axes (high frequency process). The analysis of the dielectric increments $\varepsilon_{\mathrm{s}}-\varepsilon_{\infty}$ corresponding to both relaxation processes in the isotropic phase of 6DBT allowed to estimate the dipole moment $(\mu=4.1 \mathrm{D})$ and the angle $\beta$ it forms with the long axis $\left(31^{\circ}\right)$. At the Is-SmA phase transition a considerable drop in the permittivity was observed (from ca. 9.2 to ca. 5.8). This was undoubtedly caused by a lack of orientation of the SmA phase. In the present study the static permittivity $\varepsilon_{\mathrm{s}}$ within the smectic phase reached values comparable with those observed in the isotropic phase (Figure 2). We checked, however, that the electric field of ca. $300 \mathrm{~V} / \mathrm{cm}$ did not influence the $\varepsilon_{\mathrm{s}}$-values. One can conclude therefore that the present experimental conditions facilitated the orientation of the sample parallel to the measuring field. Decreasing $\varepsilon_{\mathrm{s}}$ with lowering of the pressure is a typical behaviour observed for all nematics hitherto studied by us $[3,9,10]$.

The fits of the Debye equation to the spectra give large values of the "infinity" permittivity $\varepsilon_{\infty} \approx 5.6$, markedly exceeding the refractive index squared (see Figure 2). This means that yet another relaxation process at much higher frequencies should be observed, similarly to the studies at ambient pressure [7, 8]. In spite of the large difference in the permittivities in both experiments, mentined above, it seems wothwhile to notice the nice consistency between the present results for the relaxation times and activation enthalpy and those obtained at ambient pressure studies [7] (compare Figs. 3 and 5).

The activation parameters, $\Delta^{\#} V_{\|}$and $\Delta^{\#} H_{\|}$, obtained for 6DBT are compared in Figs. 4 and 5 with those characterizing the cyano compound $8 \mathrm{CB}$. The striking features of these plots are (i) the practically constant values of both parameters in the case of the isothiocyanato compound, having the $\mathrm{Sm} \mathrm{A}_{1}$ phase, and (ii) the marked decrease of the parameters (especially the activation volume) in the case of the cyano compound with the $\mathrm{Sm} \mathrm{A} \mathrm{A}_{d}$ phase. Therefore, the conclusion that the pressure destabilizes the antiparallel dipole-dipole associations in the Sm $A_{d}$ phase seems to be corroborated by the results of the present study.

\section{Acknowledgements}

Financial supports of the Polish Government (KBN) Grant No 2 PO3B 05913 and the Deutsche Forschungsgemeinschaft are gratefully acknowledged. 
[1] T. Brückert, S. Urban, and A. Würflinger, Ber. Bunsenges. Phys. Chem. 100, 1133 (1996).

[2] P. Markwick, S. Urban, and A. Würflinger, Z. Naturforsch. 54a, 275 (1999).

[3] S. Urban and A. Würflinger, Adv. Chem. Phys. 98, 143 (1997).

[4] A. J. Leadbetter, R. M. Richardson, and C. N. Colling, J. Physique Coll. C1, 36, 37 (1975)

[5] R. Dạbrowski and K. Czupryński, in Modern Topics in Liquid Crystals, ed. by A. Buka, World Scientific, Singapore 1993, pp. 125-169.
[6] R. Dạbrowski, B. Ważyńska, and B. Sosnowksa, Liq. Cryst. 1, 415 (1986).

[7] S. Urban, B. Gestblom, and R. Dąbrowski, Liq. Cryst 24, 681 (1998).

[8] R. Dąbrowski and S. Urban, Liq. Cryst. 24, 583 (1998).

[9] S. Urban, D. Büsing, A. Würflinger, and B. Gestblom, Liq. Cryst. 25, 253 (1998).

[10] S. Urban, Z. Naturforsch. 54a, 365 (1999). 